\title{
COMPARISON BETWEEN LASER SCANNING AND AUTOMATED 3D MODELLING TECHNIQUES TO RECONSTRUCT COMPLEX AND EXTENSIVE CULTURAL HERITAGE AREAS.
}

\author{
F. Fassi ${ }^{\text {a }}$ *, L. Fregonese ${ }^{\text {a }}$, S. Ackermann ${ }^{\text {a }}$, V. De Troia ${ }^{\text {a }}$ \\ ${ }^{a}$ Dept. of Building Environment Science \& Technology, Politecnico di Milano, 20133, Milan, Italy \\ (francesco.fassi, luigi.fregonese. sebastiano.ackermann, vincenzo.detroia)@polimi.it
}

Commission V, WG V/4

KEY WORDS: Close Range Photogrammetry, Laser scanning, Cultural Heritage, Automated 3D modelling techniques

\begin{abstract}
:
In Cultural Heritage field, the necessity to survey objects in a fast manner, with the ability to repeat the measurements several times for deformation or degradation monitoring purposes, is increasing. In this paper, two significant cases, an architectonical one and an archaeological one, are presented. Due to different reasons and emergency situations, the finding of the optimal solution to enable quick and well-timed survey for a complete digital reconstruction of the object is required.

In both cases, two survey methods have been tested and used: a laser scanning approach that allows to obtain high-resolution and complete scans within a short time and a photogrammetric one that allows the three-dimensional reconstruction of the object from images. In the last months, several methodologies, including free or low cost techniques, have arisen. These kinds of software allow the fully automatically three-dimensional reconstruction of objects from images, giving back a dense point cloud and, in some case, a surfaced mesh model.

In this paper some comparisons between the two methodologies above mentioned are presented, using the example of some real cases of study. The surveys have been performed by employing both photogrammetry and laser scanner techniques. The methodological operational choices, depending on the required goal, the difficulties encountered during the survey with these methods, the execution time (that is the key parameter), and finally the obtained results, are fully described and examinated. On the final 3D model, an analytical comparison has been made, to analyse the differences, the tolerances, the possibility of accuracy improvement and the future developments.
\end{abstract}

\section{INTRODUCTION}

In the field of Cultural Heritage, a sort of revolution about surveying and representing is coming out. Indeed, 3D measure technologies are becoming much more requested in fields such as architecture and archaeology, in which the 2D representation has been often predominant with respect to the 3D one.

Important changes has been reported on the "measuring side" of the global survey process, mainly due to the innovation of the measure technologies registered in the last years. Recently, laser scanners have become increasingly efficient in terms of point acquisition speed, portability, user friendly and cost. In addition, many current software allow to navigate huge point clouds and to make accurate measurements in real time without any additional post-elaboration even by using consumer category PC configuration. New developments and innovations can be highlighted about close-range photogrammetry elaboration processes, thanks to the introduction of new cheap and/or free software and algorithms that allow to process the data in an autonomous and user friendly mode. In addition, they promise accurate and complete results using normal (not-metric) cameras, paying less attention to the camera calibration step. These kinds of software allow the fully autonomous threedimensional reconstruction of objects from images, providing automatic tie-point detection for exterior orientation parameters computing and giving back, without any operator intervention, a dense image-matching type point cloud and, in some cases, the textured surface mesh model. All these features make these software easy to use and have the potentiality to considerably increase the use of image-based 3D reconstruction techniques in the field of cultural heritage.

Automatic image orientation and point extraction algorithms come mainly from the computer vision field: actually, they are, de facto, a kind of hybrid between specific photogrammetric software and powerful automatic modelling package. However, even if they provide attractive results, it is rarely possible to evaluate numerically the accuracy of the reconstruction unlike in photogrammetry.

The tests discussed in the present paper concern the potential accuracy obtainable from these kind of computer vision-based algorithms, focalizing attention to the precision and space accuracy validation.

\subsection{The survey methods}

Laser scanning and image-based modelling techniques have some common characteristics that brought them to be very popular in the world of cultural heritage in the last few years:

- they can be used by employing basic hardware instrumentation and user-friendly software;

- they allow to use time saving procedures for the survey and the elaboration phase as well;

- they can provide a complete 3D virtual model of the surveyed object in a short time, depending on the complexity of the object;

- they give the possibility to perform 3D measurements, to extract several type of typical 2D drawings and orthoimages;

- they allow to use the same 3D model also for visualization, documentation and distribution purposes.

In case of special urgency situations, when the survey and 3D reconstruction time is a key factor, this kind of approach become more and more "a must do". 
The aim of this paper is to investigate the performance of one of the new image-based and fully automatic software available, Agisoft Photoscan, providing a procedural and numerical comparison between the results obtained using it and the ones obtained using the laser scanning technique. Two cases of studies are described: they are the real examples of the cases in which the time is the main target to follow, as there is the need to measure, elaborate the data, extract results and make decision in the shortest possible time.

\subsection{The test cases}

In the first example, the survey method of a vast and complex archaeological findings, discovered during the construction of the underground station "Municipio" in Naples, is described. This archaeological site (about $1850 \mathrm{~m}^{2}$ just for the area aim of the survey), has a great historical relevance. However, due to its interference with the prosecution of civil engineering works, it must be partially destroyed or removed to allow the prosecution of the underground construction. In this situation, it is fundamental to speed up the acquisition phase to avoid the halt of the excavation operations and to produce the final result as soon as possible for an immediate evaluation. The survey operations should produce the classical archaeological representations in order to study the findings (orthophotos, profiles and sections) but also a detailed and accurate 3D model that would allow the eventual disassembly and re-building of the objects in the future, once the underground station construction will be accomplished. A complete ortho-image (1:50 scale) of the $3 / 4$ of the area is periodically requested (every 4-5 days) in order to show the excavation steps and compare the different stratigraphic units. Moreover, detailed ortho-images (from 1:20 to 1:5 scale) of every walls and rooms pavements present in the remaining part of the whole area are also requested. Once the ortho-images have been produced and georeferenced, they can be completed with the addition of walls silhouette, vertical profiles and plans. In the present archaeological site, the excavated parts, especially the walls, cannot be always entirely surveyed in a single survey session. Indeed, for technical reasons tied to the underground construction prosecution, most of them must be progressively destroyed or unmounted before continuing the excavation and arriving at the base of the wall. For this reason, georeferentiation is an important step, because each survey session must be reconnected to the previous one and/or to the future one, in order to reconstruct, at the end, the different layers of whole complex in its entirety and to study the modification over the centuries of the whole area.

The second test case described is the survey of the façade of the Saint Erasmo church located in Governolo (in the province of Mantua), that has been damaged subsequently to the last earthquake in Emilia region. In this kind of situations, it is important to intervene with immediate operations in order to repair the damages as soon as possible and to monitor the deterioration over time. The key aspects are the timeliness of intervention, which means the immediacy of the survey and the restitution in order to facilitate the subsequent decision-making phase.

A third example is also reported. A real test calibration site has been created ad hoc in order to better comprehend the performance of the automatic hybrid photogrammetric system in relation with laser scanning and topographic acquisition: the Mantegna Chapel in Saint Andrew Cathedral in Mantua.

\subsection{Survey and data processing technologies: some consideration}

1.3.1 Laser Scanning: Laser scanning acquisition technology has been widely discussed in the literature. As is common knowledge, it provides very quickly a dense discrete point-cloud type model of the surveyed object. The big limit of this approach, aside the instrument cost, was always the visualization and the management of a huge amount of data and the post elaboration processes time consuming required to assemble and extract a manageable 3D model. With the current hardware and software performances, a dense point-cloud model can be easily analysed without further processing (e.g. point-cloud decimation) that were necessary for measurement and analysis purposes of the object. In fact, a point cloud can now be so dense that it can be considered very similar to a continuous surfaced model and directly used for the extraction of any kind of geometric information and hence as support for immediate decision making activities. The achievable accuracy cannot be determined with certainty because the final precision of a scan depends on several factors such as inherent capabilities of the instrument, distance of the object from the scanner, angle of incidence of the laser ray with reference to the surface, materials of the object surface etc.. In addition, postelaboration procedures as the registration of different point clouds, the filtering and the meshing step, could produce unpredictable and unknown errors on the final model. The only way to evaluate correctly the final accuracy would be to compare it with a perfect reference object, which is not available in the majority of cases.

The direct use of the original acquired point cloud considerably eliminates this kind of problems. By considering the measure precision of a terrestrial laser scanner similar to the nominal accuracy of the instrument, the tolerance allowed by the scale of architectural representation (generally higher than the usual archaeological representation standards) is respected. For this reason, the laser scanner point clouds have been considered as reference model in the presented numerical comparison.

1.3.2 Automatic image based approach: In the last 10 years, the use of images to reconstruct a 3D model of an object is widely grown up. The main development regards the automatism introduced in the image matching algorithms, both for image orientation and DSM extraction. Moreover, the actual availability of a higher computational power allows to orient huge sets of photos with a free capture network and to model very complex objects.

Photogrammetry has been traditionally considered as a relatively skill intensive measuring tool characterized by a rapid data acquisition phase but followed by a time consuming manual measurement and computational data processing stage. The finding of different solutions to speed up and automatize the elaboration process are not a new task in photogrammetry. Several researches have been conducted with optimal results in industrial application since the early 1990's. Just in the 1988, many discussion were engaged about real time or near-real time photogrammetry and about the achievable accuracy (Gustafson P. C., 1988). The automatism was possible only with the use of targets that could be simply recognised by the system and used as tie point in the photogrammetric process (Remondino, 2012). The first real improvement in the automation of the orientation process came out some years later in aerial photogrammetry, by introducing the first algorithms of image matching capable to automatically detect tie-point between stereoscopic images: the system required an a-priori raw exterior orientation (even manually) among the images in order to facilitate the software to automatically find homologous tie-point between predefined 
images pairs. Anyhow, just few points could be collected and the process required the manual intervention of the operator to erase outliers and false matching points.

The effects of this limitation are much more evident in architectonic and archaeological fields, and several research groups are still working on this task. The main reason of this delay with respect to the industrial and aerial photogrammetry can be found in the intrinsic characteristic of a cultural heritage objects. Indeed, they usually are very complex 3D objects, often characterized by great extensions and big dimensions. So, a large number of photos are usually required in order to survey the whole object. However, in many cases, the use of targets is impossible or not allowed, due to the areas extension as well as to the impossibility to physically touch the object. In these situations, the availability of an automatic photogrammetric algorithm, capable to elaborate huge blocks of images and recognize automatically only natural point of the scene, would be a key solution. Moreover, the nature of the photogrammetric measurement tasks must be considered: architecture and archaeology are more demanding than industry and cartography sectors in terms of required results. Indeed, industrial application goal is generally the precise three-dimensional coordinate measurement of few points, while the aerial photogrammetry should provide accurate DSM of a territory, but for the nature of the object, it is classically a 2.5D surface model that doesn't require a great resolution.

At the contrary, when an architectonic or archaeological object must be described, a full 3D detailed DSM has to be extracted from images. These kind of objects are usually characterised by undercut areas, sharp edges, high level of detail to be represented and deep-rooted morphological diversity. This means that many series of complex and free photographic blocks are required to completely describe objects like those allaround visible. Every block can be composed by a large number of images that need to be oriented by detecting a huge amount of tie points among the images. This operation is practically impossible to perform neither manually nor by using image matching algorithms usually employed in aerial photogrammetry. Even if some tests of the past years showed that was possible to extract DSM of artistic objects using such kind of software (e.g. Apex, SocetSet, LPS), a limited number of images, an accurate intervention of the operator, a support of a good number of GCP, a classical capture geometry and a longtime elaboration were always requested (Fassi, 2007). So, when complex object must be surveyed, the use of autonomous systems that reduce as much as possible the user interaction would be a must. The new below described image based software seems to match this requisite.

\subsection{The comparison criteria}

In order to test these kind of automated image orientation and matching packages, different approaches and comparisons can be followed. In this paper, the aim is to focus on one of this kind of software: Agisosoft Photoscan. It is a complete package able to perform, with minimal external intervention (as the quality parameters setting), the whole workflow from the image orientation to the textured model extraction up to the orthophoto exporting. In this sense, it provides in a fully automatic way the final result, ready to be employed in the field of cultural heritage. For the above described reason, this software appears as a complete and easy-to-use suite that allow to quickly reconstruct 3D models.

It is able to orient a huge number of high resolution images, allowing to create, if necessary, separated capture blocks (named "Chunks") that can be aligned and merged in a second time. The possibility to add control point coordinates in the bundle and eventually fix external and interior orientation parameters, assimilates the software to the typical photogrammetric workflow. This capability allows to control the system using check points directly in the process and to verify the correctness of camera parameters. Moreover, data can be obviously georeferenced. In the tests here described, high accuracy image orientation with a generic pair pre-selection modality has been chosen. A mesh surface of the surveyed object can be obtained with different detail and quality degree. Higher quality settings can be used to obtain more detailed and accurate geometry in despite of the computational time. Generally, a DSM extracted with this settings appears to be adequately regular and of good or excellent quality. However, in those cases where the model must be used in other software or for prototyping applications, it needs to be refined to remove mesh defects. For the following cases, a middle resolution is applied, choosing 20 million faces as limit number for the mesh extraction. Then, it is used "as is" in the comparison without any further elaboration.

The main goal of this quality test is the evaluation of the accuracy of the final 3D DSM and, in one case, of the extracted orthophoto.

The workflow for the comparisons consist of three steps:

1. a complete automatic pure photogrammetric process of the images, where the scale is assigned at the and using a measured distance. 3 or 4 points collected from the laser scans are also used scale and georeference the photogrammetric model in order to perform the comparison;

2. within the photogrammetric data processing, some GCPs are given in the bundle adjustment. These points are collected by using targets visible on the scans. This type of comparison allow to study the behaviour of the photogrammetric software adding external information in relation to the complete process of the first step above described;

3. a comparison similar to the one described in the point 2 has been performed by collecting the GCPs with a total station, in order to understand the role of topography inside an automatic expeditious process.

For these tests, two professional DSLR cameras with fixed focal lenses have been employed: a Canon EOS 5D Mark II and Canon EOS 5D Mark III, equipped with a full-frame sensor of 21 and 22.3 megapixels respectively. The original fullresolution images have been used without any pre-elaboration. For the scans, a HDS7000 Leica Geosystems laser scanner has been employed.

\section{DATA, RESULTS AND COMPARISONS}

\subsection{The Naples underground findings - The case of roman thermal ruins}

2.1.1 The whole complex: The surveyed area has an extension of approximately $500 \mathrm{~m}^{2}$. Besides the survey of each single walls' façades of the complex, a global survey of the entire area, by using a bird's eye view, was requested. A remotely controlled Canon 5D Mark II camera was mounted on a horizontally movable aluminium frame controlled in one direction by a sort of "clothesline" and in a second direction by a movable roof previously positioned on two rails to preserve the ruins during the excavation progress. That solution, besides its cheapness, turned up to be a good resource to overcome the difficulties due to the height of the structures with reference to their bases. Indeed, the use of hanging baskets or UAVs was not possible due to the presence of several obstructions close to the walls. 
The ruins have a mix of high walls (circa 4 meters) and low and nested structures. For this reason, no terrestrial approach or low altitude photography could cover the areas and overcome the different image scale.

A large number of images (about 500 images) with great overlapping (about 85\%) were taken to cover the entire area in order to capture both the details at the base (far from the camera) and on the crests of the walls (close to the camera) at the same time. Moreover, for each strip, the images were taken with a convergent camera orientation, about $40^{\circ}$, along the main course direction of the camera, both forward and backward with respect to the nadiral direction and right or left just if necessary. The laser scans were performed by using an elevated number of scans at high resolution with the double goal to cover all the area and to capture the particulars of the walls, due to the necessity to extract detailed profiles and to identify each single brick or stone composing the walls.

To test the methods, two type of elaboration where conducted: the first one by processing the images "as-is", while in the second one by manually adding 18 tie-points and 7 control points in the bundle to better connect some areas with big height difference, low texture or poor quality images. In the first case, 5 points were used to scale the model and georeference it in the global coordinate system. In both cases, the points were collected manually on the laser scans by using some targets positioned on the scene for other purposes and well distinguishable natural points as well.

The 3D model was computed in low resolution, obtaining a mesh model with about 30 million of faces. This choice was mainly followed to speed up the process but also because the main goal was to produce an ortho-image of the area at 1:50 scale. Even if the low resolution of the model was enough for the requested tolerance, the process, besides the elaboration time, would potentially be able to produce a model with the same resolution of the laser scanning one.

The two methods above described gave back very similar results. At the end, the human intervention in terms of tie points addition was practically irrelevant and unnecessary. In fact, the algorithm was able to collect enough matched points even with poor quality images and to give back a robust result.

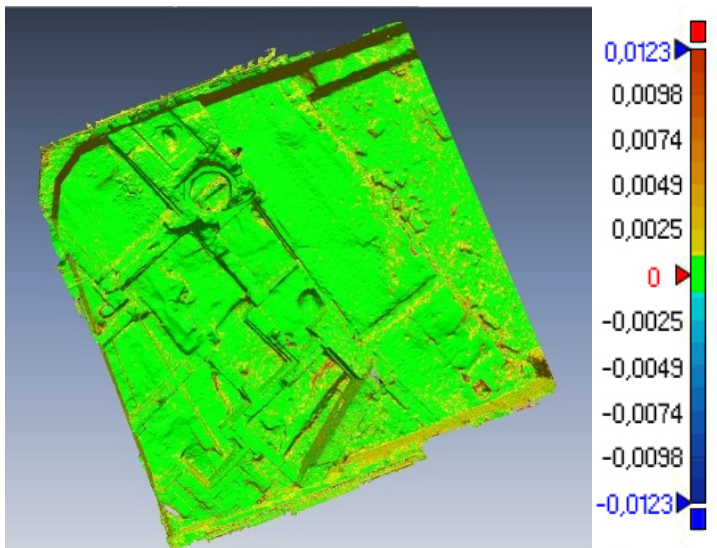

Figure 1. Comparison between the two photogrammetric models: the green areas represent a max deviation lower than 1 $\mathrm{mm}$.

By registering the two models with a surface matching algorithm (RapidformXO fine registration tool was used for the test), a max deviation lower than $1 \mathrm{~mm}$ (green areas in Figure 1) and some peaks of $3 \mathrm{~mm}$ (red areas in Figure 1), mainly close to holes or edge areas, were detected. These little differences can be due to some errors in mesh generation process. Therefore, the comparison between the two models shows clearly that there is no appreciable differences between them.

The comparison between laser and photogrammetric model instead, revealed a difference lower than $1 \mathrm{~cm}$ in $85 \%$ of the areas (Figure 2). The causes can be attributed to: i) the choice of the low resolution to build the 3D model (it was enough to build the ortophoto in the requested time but it strongly simplify its geometry); ii) the construction of the global scan cloud, as the registration of several clouds were performed only by using targets without any further refinement. However, a fine registration of the clouds were not computed because they were sufficiently accurate to extract measurement at the requested scale.

A check on the final ortho-image showed a max deviation of 3 $\mathrm{mm}$ on a well distributed set of checkpoint manually extracted from the laser-scan point cloud (Figure 3)

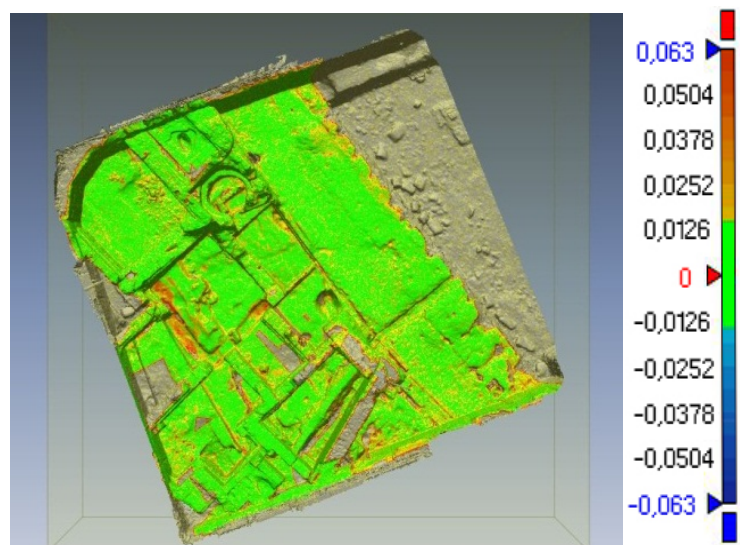

Figure 2. Comparison between Laser scanner point model and the photogrammetric one: the green areas represent a max deviation lower than $1,5 \mathrm{~cm}$.

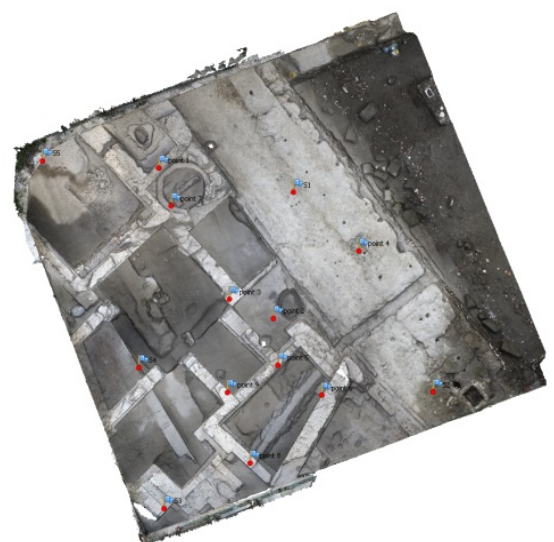

Figure 3. Checkpoints (red dots) displacement within the surveyed area

2.1.2 Wall façades. The thermal complex being surveyed consists of 50 wall façades: for each of them, an ortho-image with $1 \mathrm{~mm}$ GSD and a high resolution 3D model were requested. Furthermore, the survey and the restitution of all the façades had to be produced in a short time: the availability of an automatic and reliable data processing methodology can strongly reduce the restitution time in this sense, allowing to fulfil the imposed working time (about 1 month for the present case).

While the laser scanner based models of every façade were obtained by extrapolating them from the global point cloud, for the image-based models, each façade was treated as a single object. This choice allowed to use few images and a single 
project for the façade modelling, considerably speeding up the computational time.

For this test, a photogrammetric and a laser scanner model of a single façade were compared by following the method described in the previous section. Images were processed with Agisoft Ahotoscan without any additional information. Four natural points were manually collected from the scanner cloud to scale and georeference the façade model. In the first picture of the Figure 5, it is possible to observe that the deviation between the two models is lower than $3 \mathrm{~mm}$ (green colour) with a few peaks of about $5 \mathrm{~mm}$ (in yellow colour). This is due to the application of a rigid roto-traslation of the reference system just by manually selecting alignment points. Indeed, by performing an automatic fine registration between the two models (right picture of Figure 5), an improvement of the result was obtained, reaching more than $95 \%$ of the points with a deviation lower than $3 \mathrm{~mm}$. At the end, we can assert that the first registration allowed to obtain a sufficient result in terms of model scaling but not a perfect georeferentation between the two models (RMS=4,5 mm); however, by considering the classical architectonic scales of representation $(1: 20-1: 50)$ the result fully respects the scale tolerances.
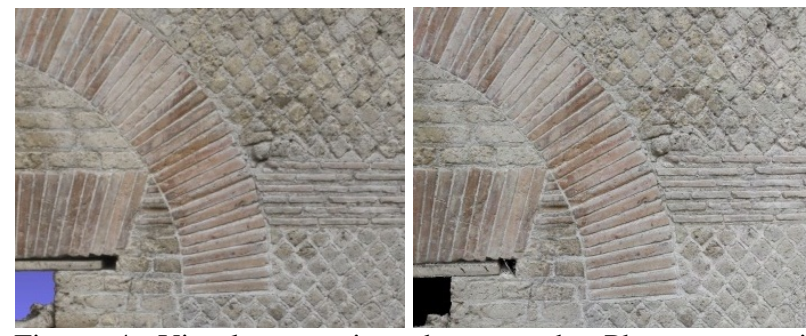

Figure 4. Visual comparison between the Photogrammetric mesh model (left) and the coloured scanner point cloud (right). It is interesting to point out that the density of the point model is so high that the difference between the laser and the photogrammetric model is almost irrelevant, allowing to clearly distinguish every details of the façade on both models

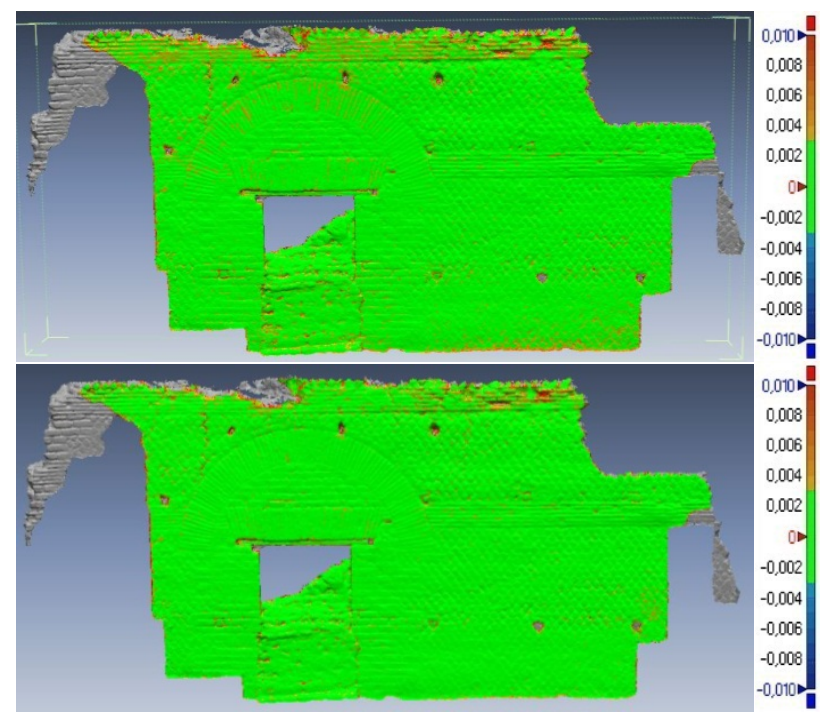

Figure 5. Comparison between image-based and laser scanner models aligned with a manual georeferentiation (above) and a fine registration (below)

From the figure 5, it is possible to notice that some part of the photogrammetric model (in grey colour) is not present in the laser scanner point one. These areas (located on the right and on the left of the main façade) are very narrow and nested compared to other structures. While it would be almost impossible to reach them with a laser scanner, the image-based survey approach, thanks to its flexibility, allowed to reach even these complex areas.

\subsection{The Sant'Erasmo church façade (Governolo)}

The Sant'Erasmo church has suffered considerable damages due to the earthquake occurred on May 2012. In particular, the upper part of the façade was heavily damaged and it could collapse in any moment. Quick measurements of about 21 façades where requested in order to organize the temporary works and make safe the structures. Plans and ortho-images where requested in this case.
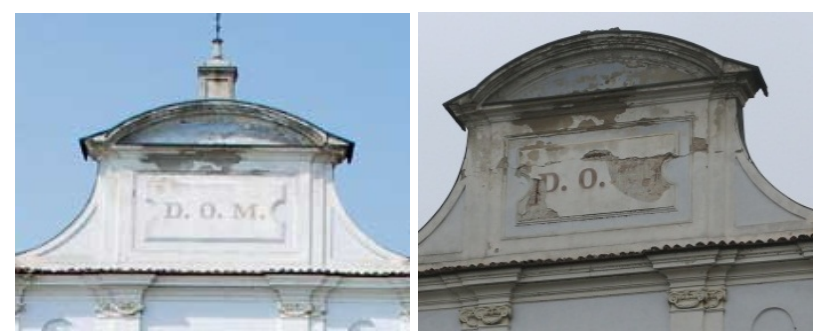

Figure 6. The upper part of the façade before (left) and after (right) the earthquake.

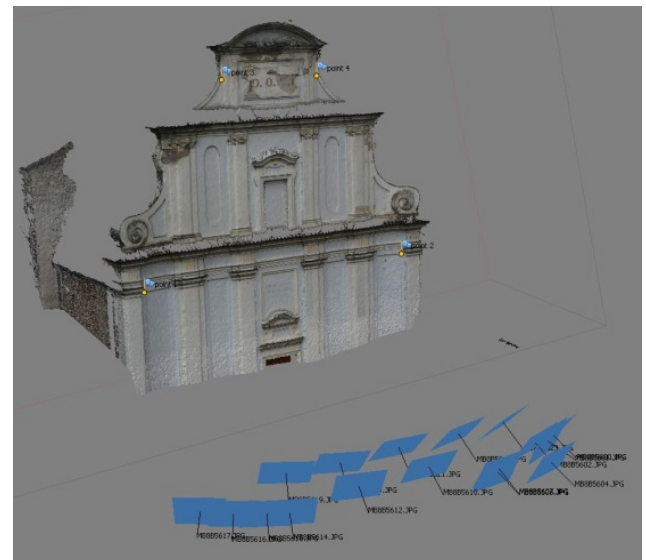

Figure 7. Photoscan high resolution model of the façade and the used camera network.

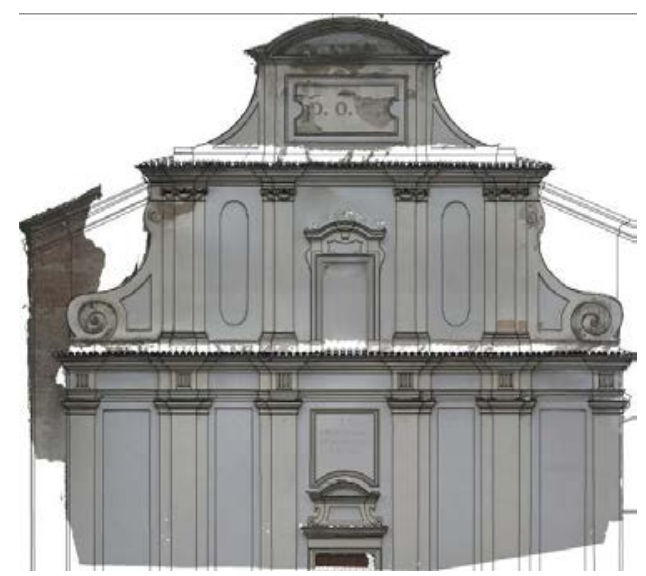

Figure 8: Comparison between the ortho-images of the façade and the digitalization obtained from the scanner point cloud.

This particular case represented a challenge because of its characteristics that would ideally preclude the use of images. Indeed, the height of the façade and the complexity of its architecture made difficult to perform a good capture geometry. 
Only few photos from the ground could be taken with almost same direction and in a quite close space in front of the façade. Moreover, the texture of the surface was very poor with the risk to heavily compromise the matching results.

Beyond expectation, the process gave an appreciable result providing a high resolution model complete and geometrically correct. The comparison with the laser scanner model gave a max deviation lower than $3 \mathrm{~mm}$ on $95 \%$ of the surface. In figure 8 , a comparison between the façade digitalization from scanner data and the orthophoto is reported: the max deviation is about 2 pixel $(4 \mathrm{~mm})$ on some decoration on the sides of the façade.

\subsection{The Mantegna chapel}

A comparative test to verify the described methods has been made by reconstructing the shape of the funeral chapel of Andrea Mantegna, located inside the Cathedral of St. Andrew in Mantua. For this analysis, the chapel has been used as a test field to perform several type of comparisons employing three different survey techniques. Even if the chapel was not injured by the earthquake, a survey has been requested in any case in order to verify its condition, as it is extremely closed to the façade of the cathedral that, at the contrary, was seriously damaged and presents a shift of some centimetres.

78 images have been acquired divided into three blocks:

1. 35 images with the optical axis horizontally disposed;

2. 25 images with the camera tilted $+45^{\circ}$ with respect to the horizontal plane;

3. 18 images with the optical axis to the zenith direction. A set of 22 coded targets for the automatic detection by the software Photoscan have been displaced on the walls. The targets have been used just for the comparison phase and have not been involved for the image orientation. The photographic acquisition has been a challenging phase due to the bad illumination condition. Even if two lamps have been used during the survey, long time exposition and high ISO have been requested in any case. As a consequence, some images could have been noisy due to the high ISO value and not completely in focus on the edges. These could have brought to the fail of the image matching process and/or to have errors in the final mesh model.
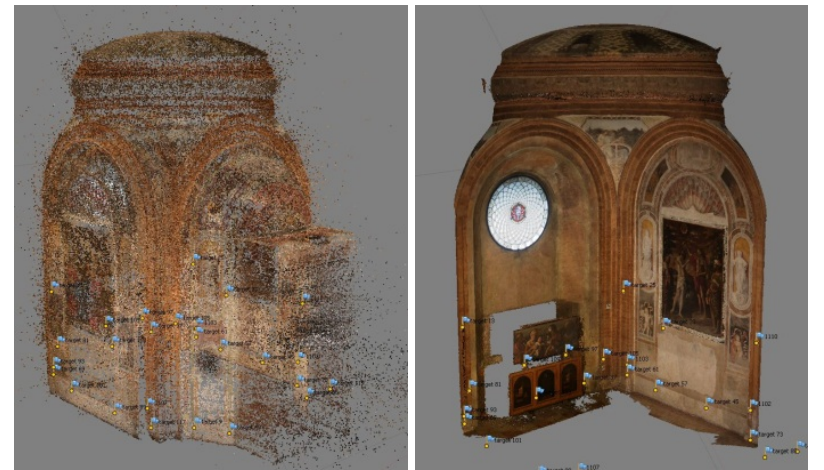

Figure 9. Tie-points cloud (left) and a cross-section of the surface model (right).

As a matter of fact, the point cloud extracted for the images orientation presented a lot of noise (figure 9 on the left) due to some bad matching. However, the extreme redundancy of the data (about 250000 3D tie points) and probably a module for self-diagnosis, allowed to produce a good final model, revealing a robust algorithm behind Agisoft Photoscan that permitted to extract reliable data even from poor quality images.

The second acquisition has been made by positioning a Leica HDS7000 scanner in the centre of the chapel in order to acquire the entire chapel with a single high resolution scan. Even in this case, a set of target has been dislocated all around to be used as a GCP (Ground Control Point) or CP (Check Point) for the comparisons. The third and last acquisition has been carried out with a total station Leica TCRM1203 from the same point of acquisition of the TLS data in order to acquire both photogrammetric and laser scanner targets.

The comparison between the laser scanner and the image-based models has confirmed the previous comparisons results, with a deviation below $3 \mathrm{~mm}$ for the $95 \%$ of the surface (Figure 10). In particular, the main errors came out due to the mesh construction and are mostly located around corners, bad illuminated areas or inside undercuts (on the vault). In this areas, the software has built a false mesh and made some mistake around open boundaries.

A further analysis on the image exterior orientation has been also performed on the 22 coded targets previously disposed, which coordinates have been measured with both laser scanner and total station. At the beginning, all the 78 images were oriented automatically in high accuracy mode with photoscan, reaching an average error of 0.679 pixel on the recognized targets. At a later stage, the exterior orientation has been performed again by including in the bundle adjustment the GCP coordinates picked on the laser point cloud. In this test, different configurations have been considered and are summarized in the Table 1 . The deviations of the camera coordinates ( $\Delta$ camera on the table 1 ) have been always carried out with respect to Mantegna 02 test case (Table 1), which is the case with minimum alignment constrain between the photogrammetric reference system and the laser one.

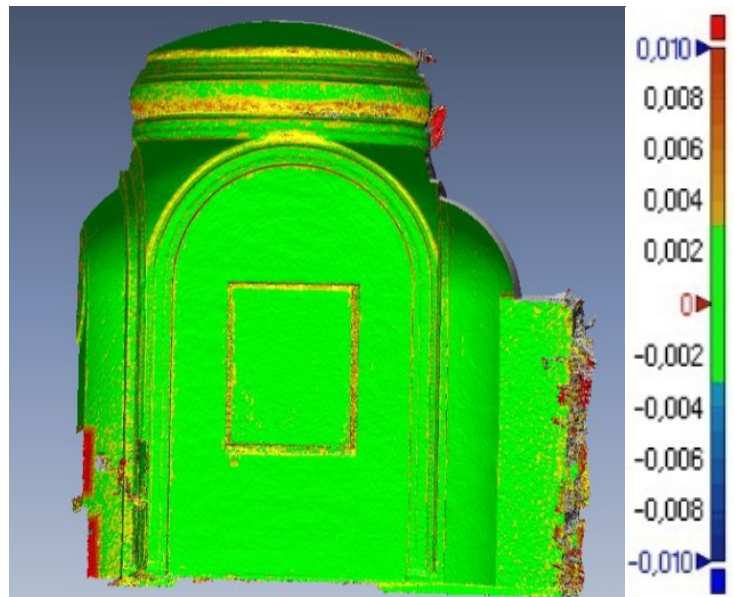

Figure 10. Comparison between laser data and photogrammetric model. In green the areas with max deviation lower than $3 \mathrm{~mm}$.

Moreover, a third image orientation has been also performed by using the same GCP which coordinates have been measured with the Total Station. The results of this test are summarized in the Table 2. Considering the distances and the architectural representation scales, the comparative tests have shown that the free photogrammetric system appears to be particularly accurate in terms of image orientation and for the subsequent definition of the photogrammetric model as well (Figure 10). The comparisons with the orientation using the GCP coordinates are reported in the RMSE values reported in Tables 1 and 2 (minimum value for Mantegna 01 equal to 0679 pixels).

As expected, there is a corresponding increase of precision in the determination of the $\sigma_{\mathrm{xyz}}$ of the $\mathrm{CP}$ when the number of GCP and their best spatial distribution are improved (Table 1 and 2).

Another consideration refers to the general comparison of the 
different values. In the case of Table 1, GCP coordinates have been manually collected from the TLS scan. It can be assumed a $\sigma_{\mathrm{xyz}}= \pm 1 \mathrm{~mm}$ in the picked laser coordinates. Similarly, the same value for the $\sigma_{\mathrm{xyz}}$ can be assumed for the topographic coordinates. At the end, the results of the comparisons are of the same order of magnitude of the input data: they provide an estimation of orientation which converges in the determination of a photogrammetric model which is congruent with the expectations. In addition, the camera calibration optimization performed with Photoscan provides results that most probably camouflage the variations of the internal parameters with external orientation shifts, as reported in the Tables 1 and 2 .

Further tests, beside the performed ones, can be taken into consideration, for example by executing comparative tests using a metric camera with known internal orientation parameters and comparing them with the estimated values obtained with Photoscan self-calibration and to analyse the impact of external orientation constraining the internal orientation parameters of the camera.

\section{CONCLUSIONS}

The achieved results illustrated that the fully automatic photogrammetric data processing approach allowed to obtain a good quality and accurate 3D model of wide as well as complex objects. The key aspect of the proposed test is the fully automatism applied for the data processing, which means a nontopographic photogrammetry with automatic point recognition without the use of targets and with a self-calibrating process by collecting a huge number of tie points.

In the presented examples, the image-based modelling methodologies gave accurate results without any external intervention. Moreover, this methodology is also low cost, flexible, quick and able to produce very accurate models, even of complex objects, comparable with 3D scanner clouds in terms of point density and accuracy. At the contrary, to extract very high resolution models, the elaboration time (even if completely autonomous) can become extremely long: this is due to the image matching software that requires much more time and very powerful hardware resources when high level details are required. From this point of view, laser scanner still remain the best technology providing full resolution scans in real time. The proposed method can be surely considered a full autonomous system, as the human intervention is required only to scale and georeference the final model, while other external data are unnecessary. Moreover, can be asserted that the method has the following properties:

- it is faster and much more accurate than manual image orientation;

- it is flexible with respect to image and camera type (close range, aerial, professional and amateur cameras);

- $\quad$ it is robust and reliable, because it also works with images of lacking quality, different scale, different illumination and poor texture (as in the case of the church in Governolo).

In addition, the module has a self-diagnosis algorithm and a manual procedure as well (check point verification) that allow to evaluate successes and failures of the computations.

Definitively, it can be considered as a good instrument for architectural and archaeological survey purposes. Indeed, it is a user friendly and fast technique that allow to extract 3D models sufficiently accurate and to perform measure within the typical archaeological and architectonical representation scale tolerances. The possibility to extract automatically "real orthophotos" is a plus as regards to similar other modules and makes it an ideal tool for professional purposes in the field of cultural heritage.

Even if the system is completely autonomous, there is a manual aspect that is essential and non-negligible: the data capture. The starting point is the necessity to perform a correct image capture geometry. In this sense, it is very difficult to give rules using this kind of image modelling modules, but some consideration, coming from experience, can be useful. The image blocks should have the right number of images: a low number of images could bring to a poor geometry and lack of data; at the contrary, too many images make the process extremely slow and it could be very difficult to find bad images if the process fails, as no control data are available to check automatic points RMS and to evaluate orientation errors. In such cases, it could be even more convenient and less time-consuming to repeat the survey and the elaboration as well. The images should have very close base capture distance in particular for the irregular areas or corners where the number of the shots must be increased. Close capture geometry and a uniform image scale usually guarantee correct camera alignment.

Additional tests will be performed in order to verify the behaviour of the module in case of little objects where the required representation scale is very big and the tolerance are more restrictive.

However, even if the "autonomous system" seems to give correct measurements from a photogrammetric point of view, it is important to remember that "as for every survey project, a testing of measures and of final restitution must always be carried out!”

\begin{tabular}{|l|c|c|c|c|c|c|c|c|c|c|}
\hline & $\begin{array}{c}\text { Optimized } \\
\text { calibration }\end{array}$ & $\begin{array}{c}\text { RMSE } \\
(\text { pixel) }\end{array}$ & $\begin{array}{c}\text { 3D GCP } \\
\text { Laser }\end{array}$ & 3D CP & $\begin{array}{c}\text { Mean GCP } \\
\text { RMS }(\mathrm{m})\end{array}$ & $\begin{array}{c}\sigma_{\text {xyz }} \text { GCP } \\
(\mathrm{m})\end{array}$ & $\begin{array}{c}\text { Mean CP } \\
\text { RMS (m) }\end{array}$ & $\begin{array}{c}\sigma_{\text {xyz }} \text { CP } \\
(\mathrm{m})\end{array}$ & $\begin{array}{c}\text { Mean } \Delta \\
\text { Camera (m) }\end{array}$ & $\begin{array}{c}\sigma_{\text {xyz }} \Delta \\
\text { Camera }(\mathrm{m})\end{array}$ \\
\hline Mantegna 01 & & 0,679 & 0 & 36 & & & & & & \\
\hline Mantegna 02 & no & 0,777 & 3 & 33 & 0,00102 & 0,00052 & 0,00236 & 0,00113 & ---- & ---- \\
\hline Mantegna 03 & yes & 0,700 & 3 & 33 & 0,00122 & 0,00079 & 0,00239 & 0,00085 & 0,00433 & 0,00688 \\
\hline Mantegna 04 & no & 0,880 & 13 & 23 & 0,00140 & 0,00058 & 0,00208 & 0,00116 & 0,00105 & 0,00057 \\
\hline Mantegna 05 & yes & 0,708 & 13 & 23 & 0,00096 & 0,00039 & 0,00165 & 0,00092 & 0,00462 & 0,00089 \\
\hline
\end{tabular}

Table 1. Resume table of the comparative test between free autonomous orientation and the orientation using TLS coordinates.

\begin{tabular}{|l|c|c|c|c|c|c|c|c|c|c|}
\hline & $\begin{array}{c}\text { Optimized } \\
\text { calibration }\end{array}$ & $\begin{array}{c}\text { RMSE } \\
\text { (pixel) }\end{array}$ & $\begin{array}{c}\text { 3D GCP } \\
\text { Laser }\end{array}$ & 3D CP & $\begin{array}{c}\text { Mean GCP } \\
\text { RMS (m) }\end{array}$ & $\begin{array}{c}\sigma_{\text {xyz }} \text { GCP } \\
(\mathrm{m})\end{array}$ & $\begin{array}{c}\text { Mean CP } \\
\text { RMS (m) }\end{array}$ & $\begin{array}{c}\sigma_{\text {xyz }} \text { CP } \\
(\mathrm{m})\end{array}$ & $\begin{array}{c}\text { Mean } \Delta \\
\text { Camera (m) }\end{array}$ & $\begin{array}{c}\sigma_{\text {xyz }} \Delta \\
\text { Camera (m) }\end{array}$ \\
\hline Mantegna 06 & no & 0.775 & 13 & 23 & 0,00151 & 0,00044 & 0,00210 & 0,00113 & ---- & --- \\
\hline Mantegna 07 & yes & 0,701 & 13 & 23 & 0,00131 & 0,00067 & 0,00172 & 0,00110 & 0,00441 & 0,00085 \\
\hline
\end{tabular}

Table 2. Resume table of the comparative test using coordinates collected with a total station (TCRA). 


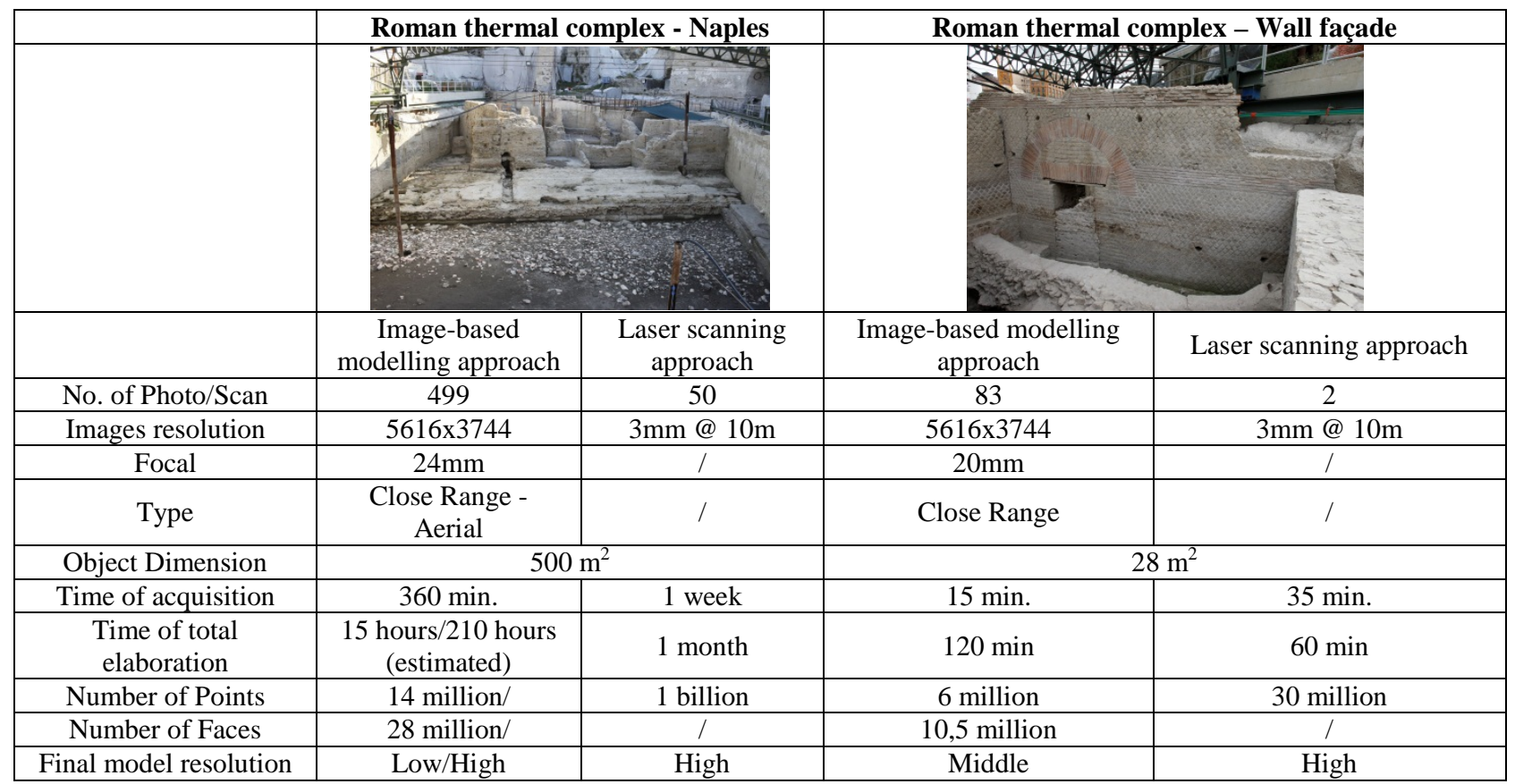

Table 3. Report of the Roman thermal complex survey in Naples and of one of its walls.

\begin{tabular}{|c|c|c|c|c|}
\hline & \multicolumn{2}{|c|}{ Sant'Erasmo Façade - Governolo } & Mantegna Chapel - Mantua \\
\hline & & & \\
& &
\end{tabular}

Table 4. Report of the Sant'Erasmo church façade and the Mantegna chapel surveys.

\section{ACKNOWLEDGEMENTS}

The authors would like to thanks Monsignor Giancarlo Manzoli, episcopal delegate for the cultural heritage of the Diocese of Mantua, Arch. Alessandro Campera, office for the ecclesiastical cultural heritage of the Diocese and Monsignor Ulisse Bresciani, vicar of the St. Andrew church, for their willingness and courtesy.

\section{REFERENCES}

Ganci, G., Handley, H., 1989. Automation in Videogrammetry. Int. Arch. Photogrammetry and Remote Sensing 32(5)

Gustafson P. C., 1988. The Application of Real-Time and Near Real-Time Photogrammetry in Industry: A Test of Accuracy ISPRS Archives - Volume XXVII Part B5.
Heipke C., 1997. Automation of interior, relative, and absolute orientation. ISPRS Journal of Photogrammetry \& Remote Sensing 52 (1997) 1-19

Fassi F., 2007. 3D modeling of complex architecture integrating different techniques - a critical overview. In: Int. Archives of Photogrammetry, Remote Sensing and Spatial Information Sciences. Zurich, Switzerland, vol. XXXVI-5/W47

Remondino F., Del Pizzo S., P. Kersten T., Troisi S., 2012. Low-Cost and Open-Source Solutions for Automated Image Orientation - A Critical Overview. In: Lecture Progress in Cultural Heritage Preservation. Notes in Computer Science Volume 7616, pp 40-54 